\title{
Mittelalterliche Augenheilkunde im Kloster St. Gallen
}

\author{
Von Peter Bischoff und Peter Speiser
}

Wahrzeichen der im Osten der Schweiz gelegenen Stadt St. Gallen ist ihr Kloster. Es geht zurück auf den heiligen Gallus, der im Jahre 612, aus Irland kommend, hier seine Einsiedlerzelle baute. Bereits im 9. Jahrhundert erreichte das Kloster eine beträchtliche Größe. Es fanden sich damals schon ein Spital und ein Ärztehaus, worin kranke Mönche und Gäste des Klosters betreut wurden. Ähnlich wie heute wurde unterschieden zwischen einem «Bettenhaus», dem eigentlichen Spital, und einer "Wachstation», der Schlafkammer für die Schwerkranken. Letztere befand sich sinnvollerweise im Ärztehaus, neben der Wohnung des Arztes. Auch eine Apotheke fehlte nicht, die ihre Medikamente vorwiegend aus dem eigenen Heilkräutergarten bezog.

Das Kloster St. Gallen war im Mittelalter als Fürstabtei das geistliche und wissenschaftliche, zeitweise auch politische Zentrum der Ostschweiz und weiter Teile Süddeutschlands. Zahlreiche wertvolle Handschriften sind uns glücklicherweise über die Säkularisation von 1805 hinweg erhalten geblieben, darunter auch medizinhistorisch interessierende. Sie werden in der berühmten Stiftsbibliothek aufbewahrt.

Aufschluß über die mittelalterliche Augenheilkunde im Kloster St. Gallen geben vor allem zwei Quellen. Zum einen finden sich in mehreren Heiligen-Viten Wunderheilungen bei Augenleiden beschrieben. Zum andern stehen uns in frühmittelalterlichen Rezeptarien Anweisungen zur Behandlung verschiedener Augenkrankheiten zur Verfügung1,2. Berichte über Wunderheilungen und Rezeptsammlungen sind medizinhistorisch vor allem interessant, weil sie Aufschluß geben über die im Mittelalter häufigsten Krankheiten und über die Versuche, sie zu heilen.

Früheste Zeugnisse über Wunderheilungen datieren aus dem 7. Jahrhundert. So wird von einer Frau berichtet, die ihre einzige, blindgeborene Tochter auf dem Rücken zum Kloster brachte. Im Kloster wurde diese Tochter durch ein Wunder sehend.

100 Jahre später wurde ein Blinder am Grab des heiligen Gallus geheilt (Abb. 1). Die Heilung wird hier sehr anschaulich geschildert: «... Da wurde ihm die Haut, die ihm vor den Augen gewachsen war, abgehauen, wie mit einem Schwert, und bald freute er sich am neu gewonnenen Lichte.» 
$i s .4$

In den pecter finer ayous ya frote und bild do withd ervon der ga fidter-ptyrcter and ticff viber lut Nond viel zistrendo veff dic erden and als er lang wiff

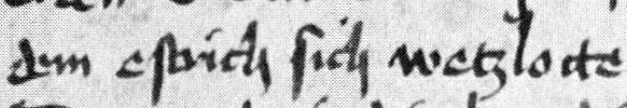
Dos nowd ion dic que dic fon ab den ayten gowadipan

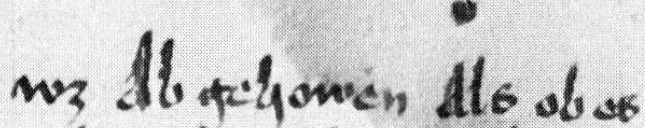
mir Amin plinet bepty etyon matr van bald neand er-enfraint vio der-wides geloungt dis vitorne hic chtere vond daw nidy ibes cutwz 3it do enplystion die

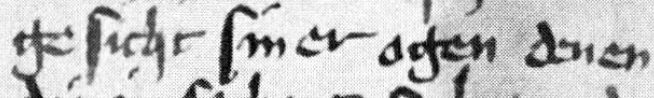
dic in fudyene po luatew-ds

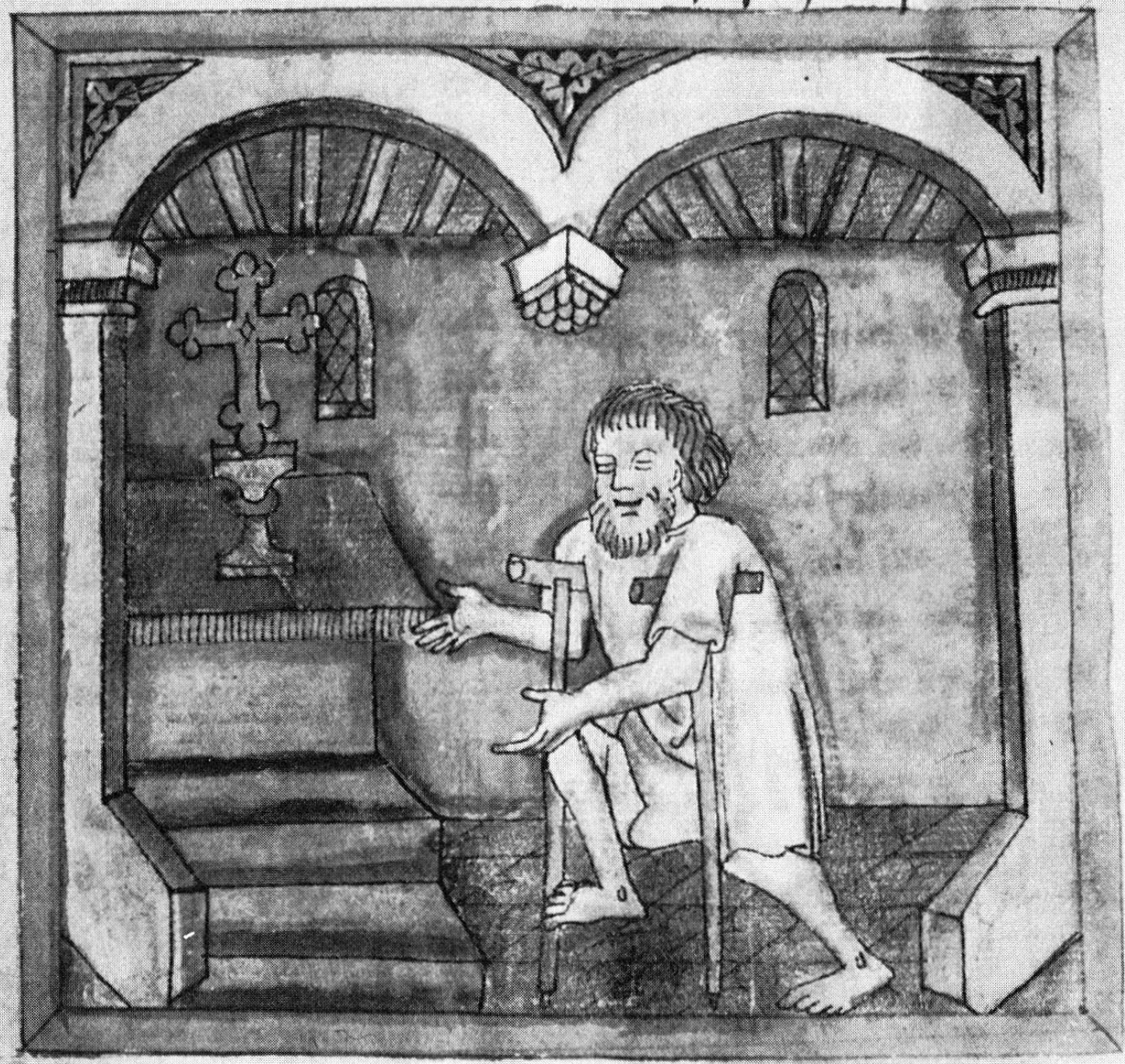

Abb. 1. Heilung eines Blinden am Gallusgrab 


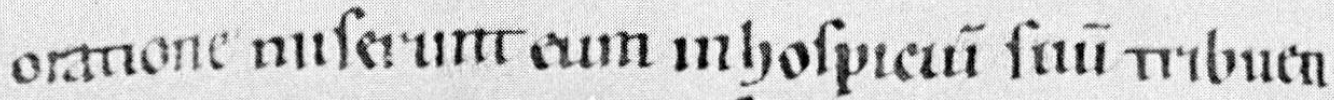

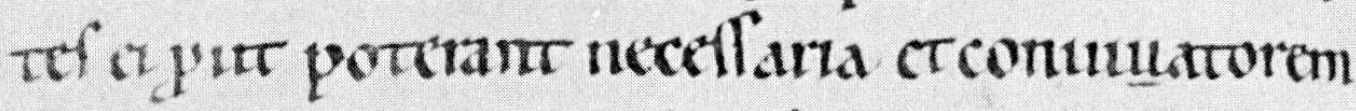
cligente' Sicq p faruf prtir pnoctanf cum ers.

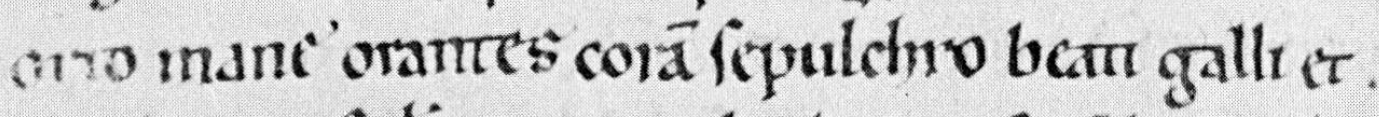

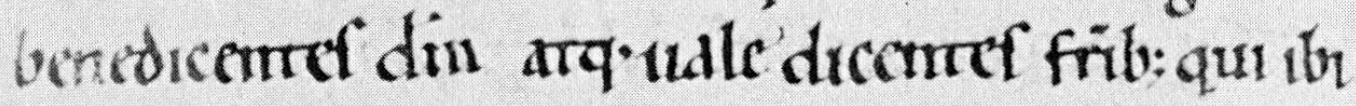

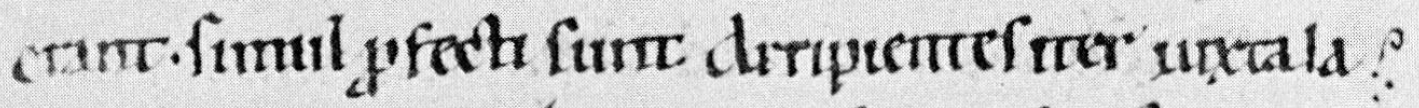

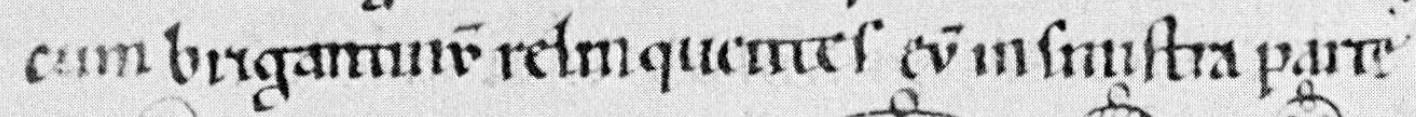

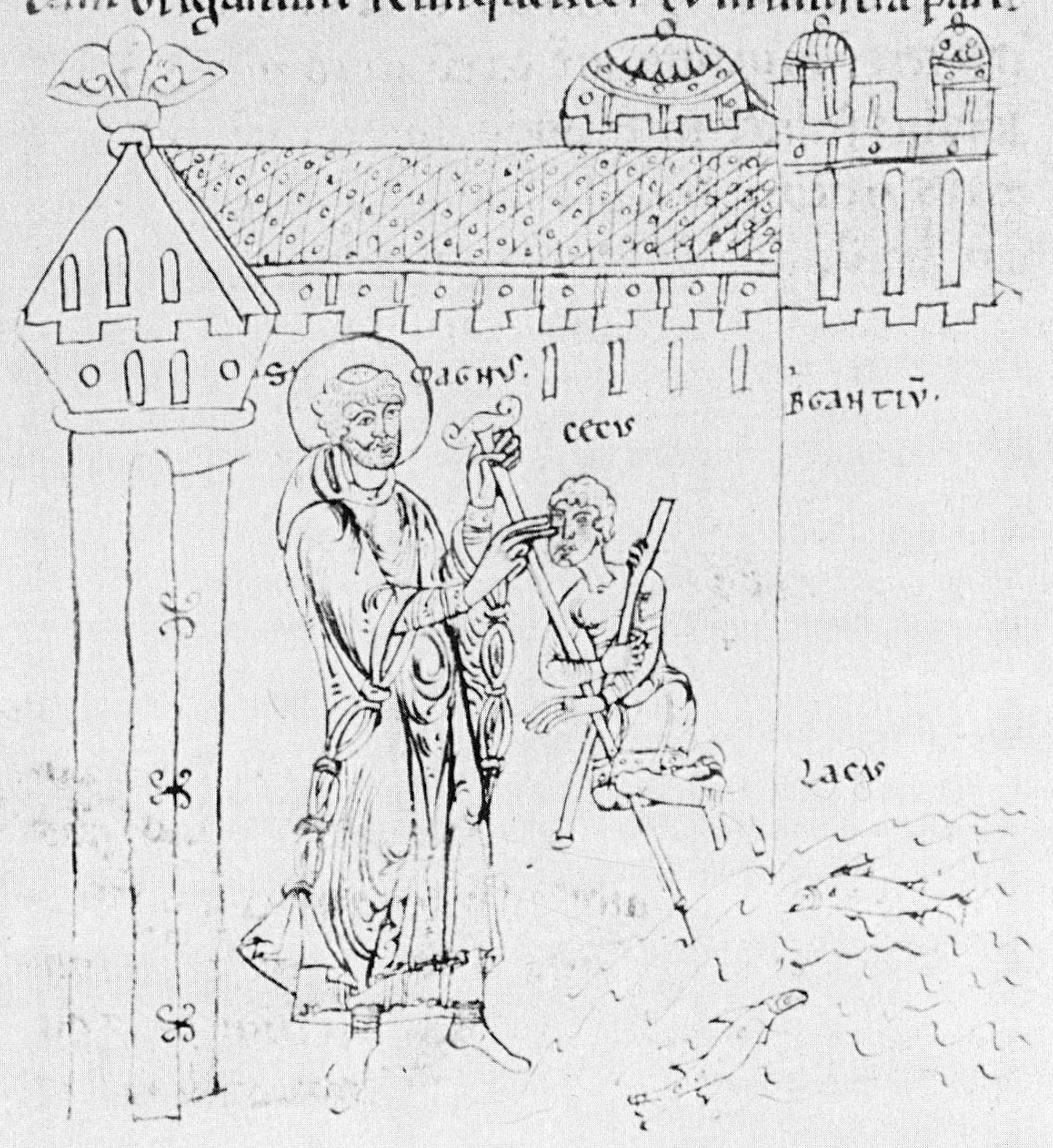

Abb.2. Heilung eines Blinden am Bodensee 
Die beiden beschriebenen Heilungen kamen ohne einen Arzt zustande. Im 8. Jahrhundert war es der heilige Magnus, der einen Blinden am Bodensee heilte (Abb.2). Laut Chronik bestrich er mit seinem Speichel das Auge des Blinden. Sogleich brach ein Strom von Blut heraus, und die Augen wurden geöffnet. - Ob man hier bereits von einem Vorgänger der modernen Vitrektomie (Entfernung des blutgefüllten Glaskörpers aus dem Auge) sprechen darf, muß allerdings offenbleiben. Die Abbildung dieser Heilung ist übrigens auch aus einem anderen Grund bemerkenswert. Es handelt sich nämlich um die früheste Darstellung des Bodensees, gezeichnet im 11. Jahrhundert. Drei Kuppeln kennzeichnen die Stadt Bregenz; der See ist unmißverständlich durch Wellen und Fische charakterisiert.

Im 10. Jahrhundert war es ein Kaufmann aus Zürich, der wegen eines schmerzhaften Augenleidens Hilfe in der Ostschweiz suchte. Eine ganz spezielle Augensalbe brachte ihn zum Sehen: «Da schabte der Arzt Märtyrerblut von der Zellenwand, legte es in ein Tüchlein und trug es mit sich ins Haus des Kranken. Und als er in sein Gemach kam, da sprengte er es in die blinden Augen. Bald erfreute sich der Kranke des neu empfangenen Lichtes und ging damit zum Grab der heiligen Magd, Gott lobend und dankend.»

Die häufigste Augenkrankheit im Mittelalter war wohl eine Entzündung der Augen, die durch Feuer und Rauch in den Wohnstätten hervorgerufen wurde. So soll etwa einem Mönch im Schlaf ein verstorbener Mitbruder erschienen sein und ihm die Vorzüge der himmlischen Wohnung geschildert haben. Der größte Vorzug liege darin, daß im Himmel kein Rauch mehr die Augen plage.

Wenn im folgenden einige Rezepte gegen Augenkrankheiten vorgestellt werden sollen, müssen deshalb Anweisungen gegen tränende Augen an erster Stelle stehen.

- AD LACRIMAS OCULORUM STRINGENDUM: Eine spezielle Augensalbe soll das Tränen zum Verschwinden bringen: «Man nehme taufrische Raute, mische sie mit Honig, Wein und Pfeffer und koche hierauf das Ganze. Mit einer Feder streiche man davon etwas in die Augen.»

- AD OCULORUM DOLORES: Leidet der Patient unter Augenschmerzen, so trinke er Aloe mit Wein. Ersteres ist ein Abführmittel, während der Wein, in genügend großer Menge genossen, die Augenschmerzen auf angenehme Art hat vergessen lassen. 
- AD OCULOS SANGUINOLENTOS: Bei blauem Auge soll man Käse und Honig aufbinden. Auch im Mittelalter wird wohl ein Lidhämatom damit innert zweier Wochen verschwunden sein.

- AD NEBULA OCULORUM: Viele Rezepte finden sich verständlicherweise gegen unscharfes Sehen, das immer auf Trübungen der vorderen Augenabschnitte zurückgeführt worden ist. Beispielsweise sollen die Augen eingesalbt werden mit Fenchelwurzelsaft und Weihrauch, zusammen mit der Asche vom verbrannten Kopf eines Tintenfisches und Honig. Damit soll «das Licht zurückkehren».

- AD OMNES VERMES ET TINEAS IN OCULO: Das Juckgefühl entzündeter Augen wurde vom Wirken kleinster «Würmer und Motten im Auge» hergeleitet. Der Arzt konnte sich mit folgendem Rezept großes Ansehen erwerben: «Es soll Schweinekot in einem grob gewobenen leinenen Tuch auf die Augen gebunden werden.» Wenn dann alsbald auf diesem schauerlichen Verband Würmer sich tummeln, so soll der gutgläubige Patient dankbar bewundern, wie rasch diese Würmer aus dem Auge vertrieben worden sind.

Im Zusammenhang mit mittelalterlicher Augenheilkunde werden vielleicht einige Ausführungen zu chirurgischen Augeneingriffen, wie etwa dem Starstechen, vermißt. Im Kloster St.Gallen sind jedoch zu keiner Zeit Augenchirurgen am Werk gewesen, retrospektiv darf man wohl sagen, zum Glück.

Abschließend ist man nach diesem kurzen Rückblick ins Mittelalter gerne versucht, zu fragen, wie wohl unsere heutige Augenheilkunde von allfälligen Nachfahren in 500 Jahren beurteilt werden könnte.

\section{Quellennachweis}

${ }^{1}$ Duft, J., Notker der Arzt. Klostermedizin und Mönchsarzt im frühmittelalterlichen St. Gallen. St. Gallen 1975.

${ }^{2}$ Jörimann, J., Frühmittelalterliche Rezeptarien, Diss. Univ. Zürich 1925. 


\section{Verdankung}

Herrn Prof. Johannes Duft, Stiftsbibliothek St. Gallen, danke ich für die freundliche Überlassung der Abbildungen.

\section{Summary}

In the famous library of the abbey of St.Gall there are numerous manuscripts of medicohistorical interest. Descriptions of miraculous cures and collections of prescriptions give information about the most frequent eye diseases in the Middle Ages and describe the attempts of their cure.

Dr. med. Peter Bischoff / Prof. Dr. med. Peter Speiser Klinik für Augenkrankheiten (Kantonsspital)

CH-9007 St. Gallen 